\title{
Structural Basis of inhibition of Insulin-Regulated Aminopeptidase by a macrocyclic peptidic inhibitor
}

Anastasia Mpakali ${ }^{1}$, Emmanuel Saridakis $^{1}$, Petros Giastas ${ }^{1,+}$, Zachary Maben $^{2}$, Lawrence J. Stern' ${ }^{2}$, Mats Larhed ${ }^{3}$, Mathias Hallberg ${ }^{3}$ and Efstratios Stratikos ${ }^{1}$.

\footnotetext{
${ }^{1}$ National Center for Scientific Research Demokritos, Agia Paraskevi, Athens 15310, Greece 2Department of Pathology, University of Massachusetts Medical School, Worcester, Massachusetts 01655, United States

${ }^{3}$ Department of Pharmaceutical Biosciences, Division of Biological Research on Drug Dependence, BMC, Uppsala University, P.O. Box 591, SE-751 24 Uppsala, Sweden
} 


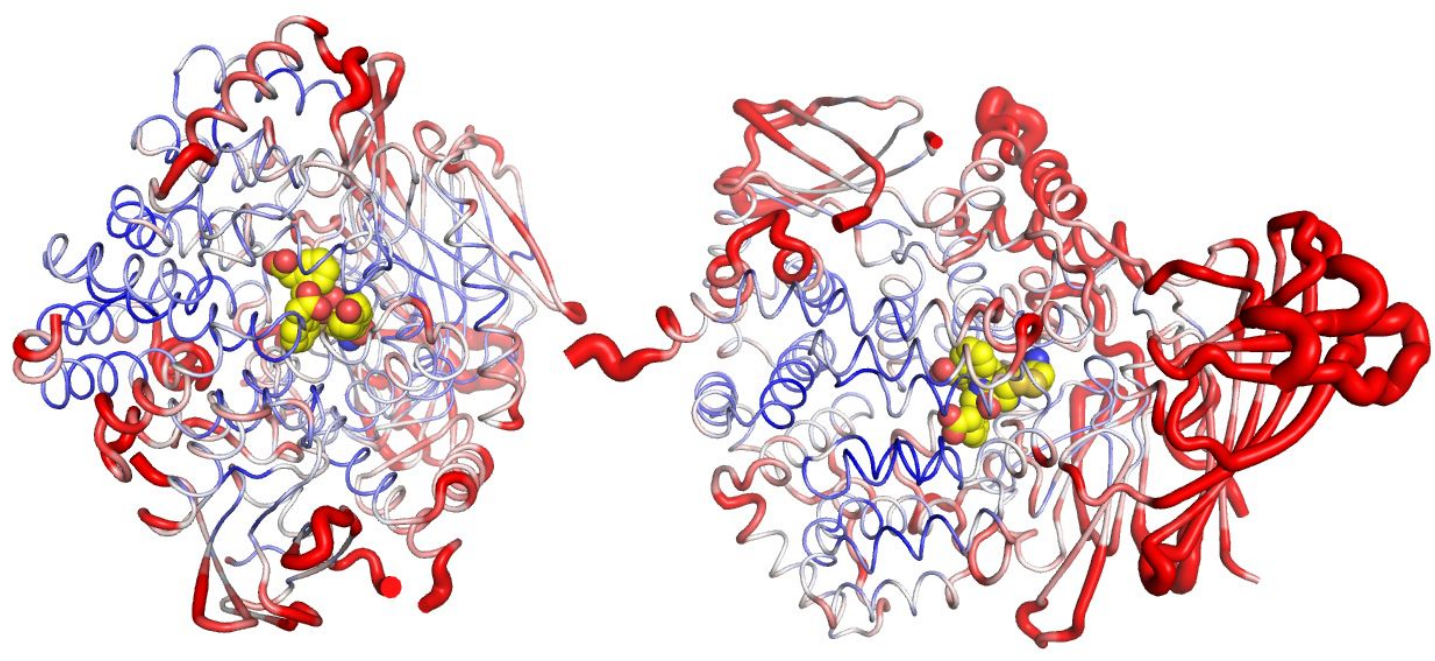

Supplemental Figure 1: Schematic representation of the crystallographic IRAP dimer colored to indicate B-factors. IRAP is shown as a ribbon whose thickness indicates relative B-factor. Inhibitor is shown in yellow spheres (oxygen=red, nitrogen=blue). Note that the region around the inhibitor has overall low B-factors compared to the rest of the protein. 


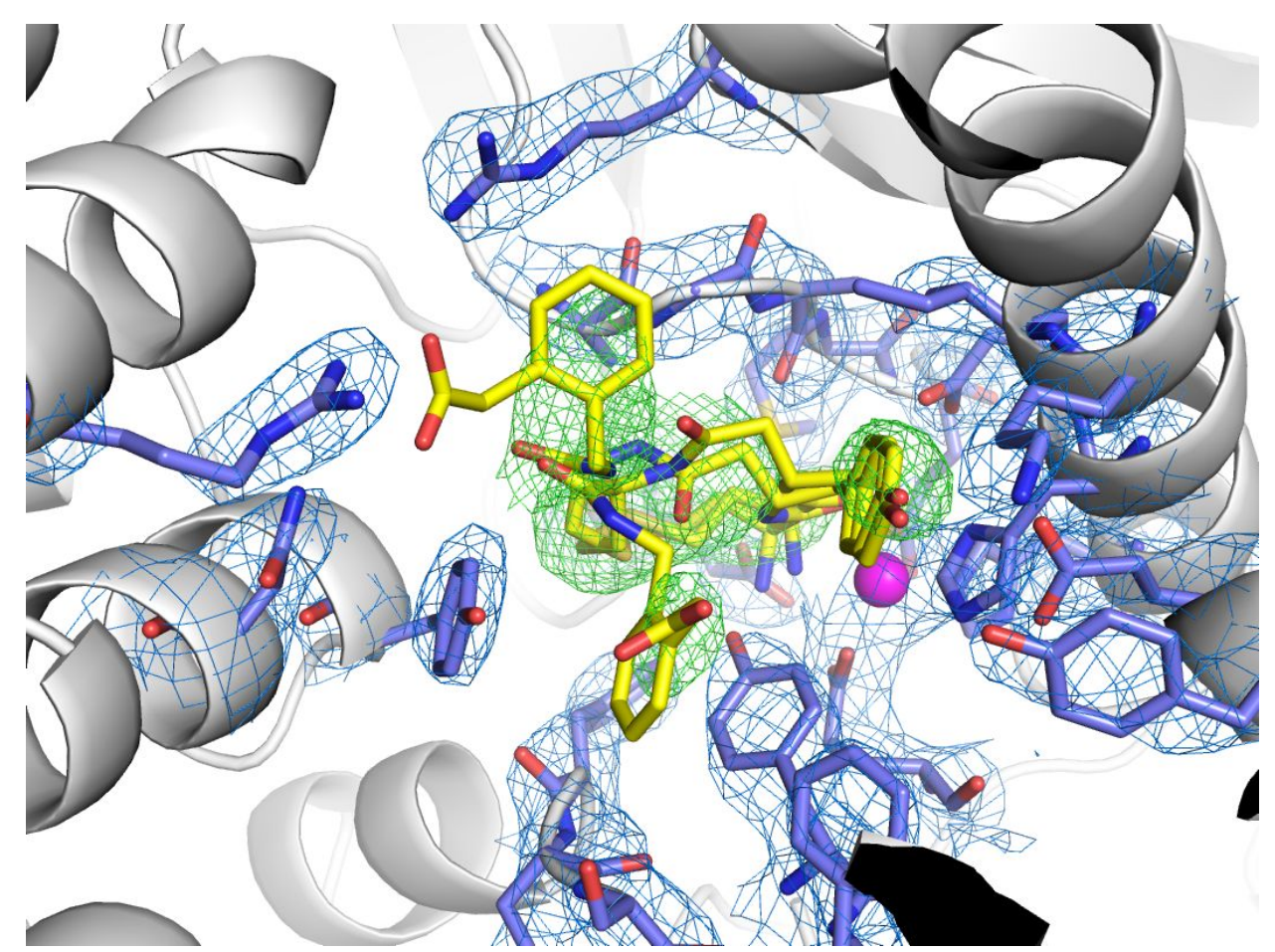

Supplemental Figure 2: Omit $F_{o}-F_{c}$ map contoured at 2 sigma of the IRAP active site shown as a green mesh. Both poses of the inhibitor are shown in stick representation (carbon=yellow, oxygen=red, nitrogen=blue, sulfur=orange). The unbiased $2 F_{o}-F_{c}$ map is shown as a blue mesh contoured at 1.5 sigma. Protein residues located within $4 \AA \AA$ of the inhibitor are shown in stick representation (carbon=cyan, nitrogen=blue, oxygen=red). The active-site zinc(II) atom is shown as a magenta sphere. Cartoon representation of the protein is shown in light grey. 


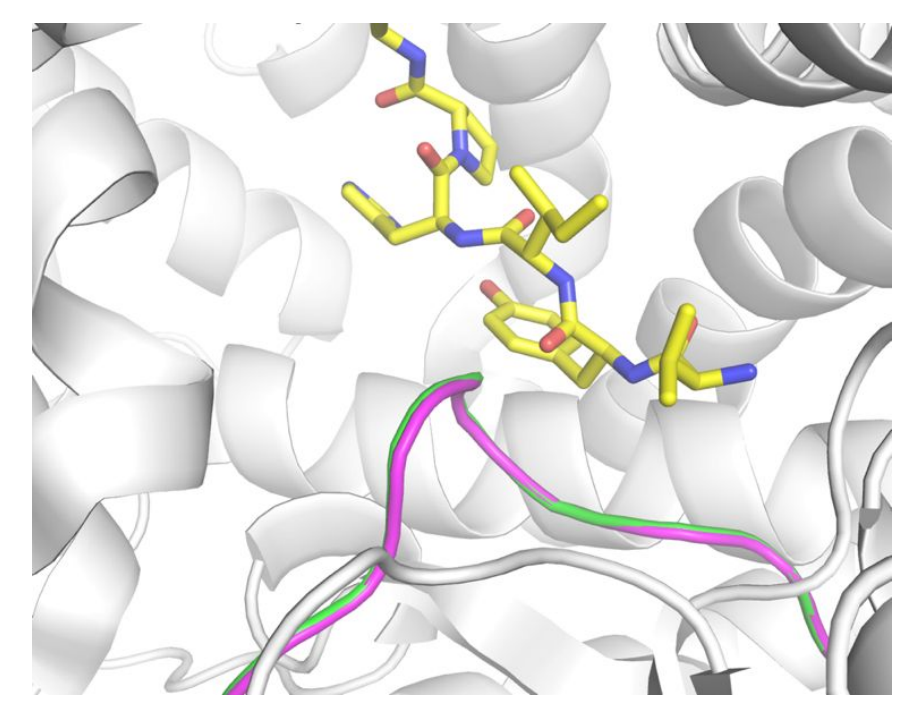

Supplemental Figure 3: Comparison of the conformation of the GAMEN loop in aminopeptidase $\mathrm{N}$ active site ${ }^{1}$. Structure is shown in the same view as the structures of IRAP in Figure 3. Two known structures of aminopeptidase $\mathrm{N}$ are superimposed (PDB codes 4FYQ and 4FYS, with no ligand bound and with AngIV bound respectively). AngIV is depicted as yellow sticks (oxygen atoms in red and nitrogen atoms in blue). The GAMEN loop is colored green for the empty structure and magenta for the AngIV-bound structure. No major differences between the conformation of the GAMEN loop are evident between the empty and inhibitor-bound structure. 
Supplemental Table 1: Data collection and refinement statistics.

\begin{tabular}{|c|c|}
\hline Wavelength $(\AA)$ & 0.97624 \\
\hline Resolution range $(\AA)$ & $45.31-3.20(3.31-3.20)$ \\
\hline Space group & $\mathrm{P} 2_{1}$ \\
\hline Unit cell & $\begin{array}{c}a=73.42 \AA b=118.63 \AA c=141.39 \AA \\
\beta=102.767^{\circ}\end{array}$ \\
\hline Total reflections & $114638(11884)$ \\
\hline Unique reflections & $38228(1690)$ \\
\hline Multiplicity & $3.0(3.1)$ \\
\hline Completeness (\%) & $81.67(43.65)$ \\
\hline Mean I/sigma(I) & $4.34(0.98)$ \\
\hline Wilson B-factor $\left(\AA^{2}\right)$ & 53.45 \\
\hline R-merge & $0.254(1.245)$ \\
\hline R-meas & $0.308(1.514)$ \\
\hline R-pim & $0.172(0.826)$ \\
\hline $\mathrm{CC} 1 / 2$ & $0.979(0.321)$ \\
\hline $\mathrm{CC}^{*}$ & $0.995(0.697)$ \\
\hline Reflections used in refinement & $32002(1690)$ \\
\hline Reflections used for R-free & $1563(90)$ \\
\hline R-work (\%) & $27.6(31.7)$ \\
\hline R-free (\%) & $31.2(39.3)$ \\
\hline CC(work) & $0.831(0.671)$ \\
\hline $\mathrm{CC}$ (free) & $0.790(0.419)$ \\
\hline Number of non-hydrogen atoms & 14298 \\
\hline macromolecules & 13695 \\
\hline ligands & 575 \\
\hline solvent & 28 \\
\hline Protein residues & 1709 \\
\hline RMS(bonds) (Å) & 0.008 \\
\hline RMS(angles) $\left({ }^{\circ}\right)$ & 1.36 \\
\hline Ramachandran favored (\%) & 95.46 \\
\hline Ramachandran allowed (\%) & 3.89 \\
\hline Ramachandran outliers (\%) & 0.65 \\
\hline Rotamer outliers (\%) & 0.00 \\
\hline Clashscore & 19.0 \\
\hline Average B-factor $\left(\AA^{2}\right)$ & 38.41 \\
\hline macromolecules $\left(\AA^{2}\right)$ & 37.73 \\
\hline ligands $\left(\AA^{2}\right)$ & 55.13 \\
\hline solvent $\left(\AA^{2}\right)$ & 26.69 \\
\hline Number of TLS groups & 1 \\
\hline
\end{tabular}

(In brackets, numbers for the highest resolution shell) 


\section{Materials and Methods}

\section{Protein expression and purification}

Expression of the soluble domain of IRAP has been described previously ${ }^{2}$. IRAP was isolated after secretion from stably-transfected HEK $293 \mathrm{~S} \mathrm{GnTI}^{(-)}$cells and purified by affinity chromatography (anti-Rho1D4 tag Ab, elution with Rho1D4 peptide) and size-exclusion chromatography (Sephacryl 200 16/60 HR column; GE Healthcare). The enzyme was concentrated using size-exclusion spin filters (Ultrafree-4) and used for crystallization trials, or aliquoted and frozen at $-80^{\circ} \mathrm{C}$ in $10 \mathrm{mM}$ Hepes $(\mathrm{pH} 7.5)$ and $150 \mathrm{mM} \mathrm{NaCl}, 10 \%$ glycerol until needed for enzymatic assays.

\section{Enzymatic assays}

The enzymatic activity of IRAP was measured by following the change in fluorescent signal produced upon digestion of the substrate L-leucine 7-amido-4-methyl coumarin or Larginine-7-amido-4-methylcoumarin (L-AMC or R-AMC, Sigma-Aldrich). The emission was set at $460 \mathrm{~nm}$, whereas the excitation was set at $380 \mathrm{~nm}$. Measurements were performed on a TECAN spark $10 \mathrm{M}$ microplate fluorescence reader as previously described ${ }^{3}$. For measurements of the $\mathrm{IC}_{50}$ value, the activity of the enzyme was measured in the presence of increasing concentrations of the inhibitor and data were fit to a 4-parameter variable slope inhibition model using Graphpad Prism. For Michaelis-Menten analysis, the enzymatic assay was performed using increasing concentrations of substrate and inhibitor and the data were fit to a Michaelis-Menten model using Graphpad Prism.

\section{Crystallization and data collection}

Crystals of IRAP complexed with the macrocyclic peptidic inhibitor HA08 were obtained by screening against the commercially available PACT-premier crystallization screen (Molecular Dimensions), since no good quality and stable crystals could be obtained from previously known IRAP crystallization conditions. The screening trials were set up using the Oryx 4 (Douglas Instruments Ltd, U.K.) crystallization robot. Crystals were grown at $16^{\circ} \mathrm{C}$ by the sitting-drop vapor-diffusion method, in $25 \%(\mathrm{w} / \mathrm{v})$ polyethylene glycol (PEG) of mean M.W 1500 and 0.1 M SPG buffer (Succinic acid, Phosphate, Glycine), pH 5.0 (condition A2 of the PACT Screen). One hour prior to dispensing the crystallization drops, purified IRAP concentrated to $5 \mathrm{mg} / \mathrm{ml}$ was mixed with the inhibitor in a molar ratio $1: 5$ and the mixture was kept at $4^{\circ} \mathrm{C}$ during that incubation period. Crystals of various morphologies and sizes were chosen for harvesting. Cryoprotection was achieved by rapid immersion in a solution containing the precipitant and $20 \%$ ethylene glycol for 5-10 seconds and the crystals were subsequently flash frozen in liquid $\mathrm{N}_{2}$. Several datasets were collected at $100 \mathrm{~K}$ at a wavelength of $0.976 \AA$ A on synchrotron radiation beamline P13 of Petra III, EMBL, Hamburg, Germany. The reflections were integrated with XDS ${ }^{4}$, the space group was determined with POINTLESS ${ }^{5}$ and data scaling and merging were performed with SCALA ${ }^{6}$ of the CCP4 suite ${ }^{7}$. The best resulting dataset displayed highly anisotropic data extending nominally to $2.6 \AA$. Data were processed with the Staraniso software ${ }^{8}$, which applied a cut-off ellipsoid with axes 3.04, 3.01 and $3.84 \AA$. The anisotropy ratio, fractional anisotropy and anisotropic signal/noise 
ratio were given as $0.463,0.230$ and 1.96 respectively. Best and worst diffraction limits after cut-off were at 3.01 and $17.24 \AA$ respectively. Completeness of observed data for the ellipsoidal cut-off shell was 0.891 ( 0.478 for the outer shell) and for the corresponding spherical shell 0.698 ( 0.164 for the outer shell). Because of the low (spherical shell) completeness and poor scaling of the highest resolution data, we decided to apply the more stringent cut-off of $3.2 \AA$ to the reflections file output by Staraniso. $4.9 \%$ of the reflections were flagged for $R_{\text {free }}$ calculations. The crystals belonged to space group $P 2_{1}$ with $a=73.42 \AA$, $b=118.63, c=141.39 \AA$, and $\beta=102.77^{\circ}$.

\section{Structure determination and refinement}

The structure was solved by molecular replacement with PHASER $^{9}$ using as a search model the closed structure of IRAP (PDB ID: 5MJ6) ${ }^{2}$. Two protein molecules were found in the asymmetric unit (referred to as chains $A$ and $B$ ). Inspection of the initial electron density maps showed clearly the presence of a circular shaped electron density in the zinc binding site region, which was attributed to HA08. Notably, the electron density showed better quality at the second molecule of the asymmetric unit (chain B). Two different conformations of ligand HAO8 were built in each chain (discussed in the main text). Structure refinement was conducted with phenix.refine ${ }^{10}$ using restrained refinement and non-crystallographic symmetry restraints, while at the final stages TLS refinement was included. Alternate cycles of model building and real space refinement were performed in $\operatorname{Coot}^{11}$. The structure converged to $R$ and $R_{\text {free }}$ of 27.6 and $31.2 \%$ respectively. In chain $A$, no density was visible before residue 157 and between residues 337-341 and 638-649, while in chain B no density was visible before residue 159 and between residues 600-601 and 638-649. In the model were also included 24 molecules of $\mathrm{N}$-acetyl-D-glucosamine, 2 molecules of beta-D-mannose, 1 succinic acid, 1 phosphate ion, 3 polyethylene glycols, 2 molecules of 1,2-ethanediol and 28 water molecules.

\section{Small-angle $x$-ray scattering}

IRAP freshly isolated by gel filtration using a Superdex 200 10/300 GL column (GE Healthcare) in $50 \mathrm{mM}$ HEPES pH 7.5, 200mM NaCl, $0.02 \%$ (w/v) $\mathrm{NaN}_{3}$ buffer was mixed with $100 \mu \mathrm{M}$ HA08 inhibitor, if indicated, and concentrated in a rinsed Centricon 10kDa MWCO $0.5 \mathrm{~mL}$ centrifugal concentrators equilibrated in the same buffer. Under these conditions the enzyme is essentially $100 \%$ inhibited. Concentrator retentate and flow-through were stored and used as sample and buffer respectively during SAXS data collection. SAXS data was collected for each sample at three concentrations $(4 \mathrm{mg} / \mathrm{mL}, 2 \mathrm{mg} / \mathrm{mL}$, and $1 \mathrm{mg} / \mathrm{mL})$. SAXS data were collected at the NSLS-II 16-ID LiX beamline ${ }^{12}$. Scattering curves from the three different concentrations were overlaid, evaluated for aggregation or repulsion in the low $q$ region, and merged into one curve for analysis. Guinier analysis of SAXS data was used to estimate the radius of gyration (Rg). Pairwise distribution analysis is a model-independent approach to characterize the distribution of interatomic distances within the scattering particle. One key parameter is the maximum of this distribution, Dmax, which corresponds the maximum interatomic distance in the particle. In this analysis, which uses a wider range of data than Guinier analysis, a similar trend is observed, with Dmax decreasing when HA08 is present. Guinier analysis was performed using AUTORG program, and pairwise distribution analysis was performed using AUTOGNOM (now known as DATGNOM), both in the ATSAS 


\section{Supplemental Material}

software package (version 2.5.2, https://www.embl-hamburg.de/biosaxs/software.html). Error bars denote errors in the linear fit. 


\section{References}

1. Wong, A. H.; Zhou, D.; Rini, J. M., The X-ray crystal structure of human aminopeptidase $\mathrm{N}$ reveals a novel dimer and the basis for peptide processing. J. Biol. Chem. 2012, 287 (44), 36804-13.

2. Mpakali, A.; Saridakis, E.; Harlos, K.; Zhao, Y.; Kokkala, P.; Georgiadis, D.; Giastas, P.; Papakyriakou, A.; Stratikos, E., Ligand-Induced Conformational Change of InsulinRegulated Aminopeptidase: Insights on Catalytic Mechanism and Active Site Plasticity. J. Med. Chem. 2017, 60 (7), 2963-2972.

3. Mpakali, A.; Saridakis, E.; Harlos, K.; Zhao, Y.; Papakyriakou, A.; Kokkala, P.; Georgiadis, D.; Stratikos, E., Crystal Structure of Insulin-Regulated Aminopeptidase with Bound Substrate Analogue Provides Insight on Antigenic Epitope Precursor Recognition and Processing. The Journal of Immunology 2015, 195 (6), 2842-2851.

4. Kabsch, W., Xds. Acta Crystallogr D Biol Crystallogr 2010, 66 (Pt 2), 125-32.

5. Evans, P. R., An introduction to data reduction: space-group determination, scaling and intensity statistics. Acta Crystallogr D Biol Crystallogr 2011, 67 (Pt 4), 282-92.

6. Evans, P., Scaling and assessment of data quality. Acta Crystallographica Section DBiological Crystallography 2006, 62, 72-82.

7. Winn, M. D.; Ballard, C. C.; Cowtan, K. D.; Dodson, E. J.; Emsley, P.; Evans, P. R.; Keegan, R. M.; Krissinel, E. B.; Leslie, A. G.; McCoy, A.; McNicholas, S. J.; Murshudov, G. N.; Pannu, N. S.; Potterton, E. A.; Powell, H. R.; Read, R. J.; Vagin, A.; Wilson, K. S., Overview of the CCP4 suite and current developments. Acta Crystallogr D Biol Crystallogr 2011, 67 (Pt 4), 235-42.

8. Tickle, I. J., Flensburg, C., Keller, P., Paciorek, W., Sharff, A., Vonrhein, C., Bricogne, G., STARANISO (http://staraniso.globalphasing.org/cgi-bin/staraniso.cgi). Cambridge, United Kingdom: Global Phasing Ltd.

9. McCoy, A. J.; Grosse-Kunstleve, R. W.; Adams, P. D.; Winn, M. D.; Storoni, L. C.; Read, R. J., Phaser crystallographic software. J. Appl. Crystallogr. 2007, 40 (Pt 4), 658-674. 10. Afonine, P. V.; Grosse-Kunstleve, R. W.; Echols, N.; Headd, J. J.; Moriarty, N. W.; Mustyakimov, M.; Terwilliger, T. C.; Urzhumtsev, A.; Zwart, P. H.; Adams, P. D., Towards automated crystallographic structure refinement with phenix.refine. Acta Crystallogr D Biol Crystallogr 2012, 68 (Pt 4), 352-67.

11. Emsley, P.; Lohkamp, B.; Scott, W. G.; Cowtan, K., Features and development of Coot. Acta Crystallogr D Biol Crystallogr 2010, 66 (Pt 4), 486-501.

12. DiFabio, J.; Chodankar, S.; Pjerov, S.; Jakoncic, J.; Lucas, M.; Krywka, C.; Graziano, V.; Yang, L., The life science $x$-ray scattering beamline at NSLS-II. AIP Conference Proceedings 2016, 1741 (030049), 4. 